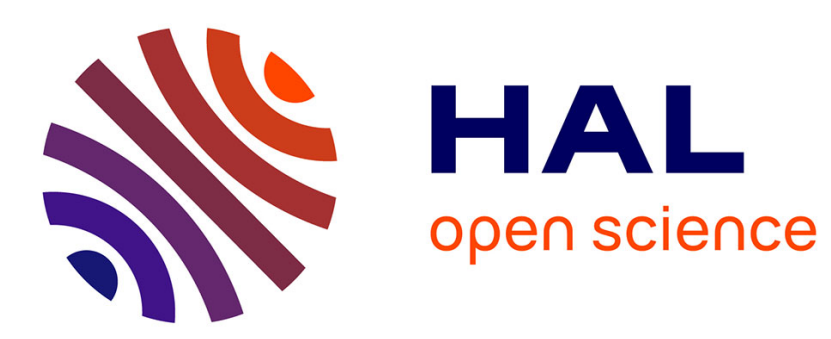

\title{
Une approche sociocritique des usages numériques en éducation
}

\author{
Simon Collin, Nicolas Guichon, Jean Gabin Ntebutse
}

\section{To cite this version:}

Simon Collin, Nicolas Guichon, Jean Gabin Ntebutse. Une approche sociocritique des usages numériques en éducation. STICEF (Sciences et Technologies de l'Information et de la Communication pour l'Éducation et la Formation), 2015, Revue STICEF, 22. hal-01218240

\author{
HAL Id: hal-01218240 \\ https://hal.science/hal-01218240
}

Submitted on 20 Oct 2015

HAL is a multi-disciplinary open access archive for the deposit and dissemination of scientific research documents, whether they are published or not. The documents may come from teaching and research institutions in France or abroad, or from public or private research centers.
L'archive ouverte pluridisciplinaire HAL, est destinée au dépôt et à la diffusion de documents scientifiques de niveau recherche, publiés ou non, émanant des établissements d'enseignement et de recherche français ou étrangers, des laboratoires publics ou privés. 


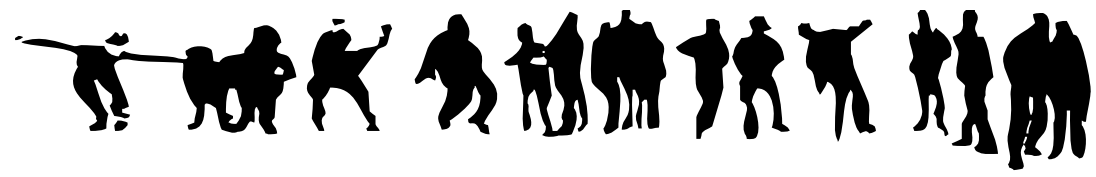

\section{Une approche sociocritique des usages numériques en éducation}

Simon COLLIN (CRIFPE, Université du Québec à Montréal), Nicolas GUICHON (ICAR, Université Lumière Lyon 2), Jean Gabin NTÉBUTSÉ (CERTA, Université de Sherbrooke)

RÉSUMÉ • Cet article a pour objectif de contribuer à formaliser une approche sociocritique du numérique en éducation. Telle que nous la concevons, cette approche consiste à étudier les relations entre le profil et le contexte socioculturel des élèves et leur disposition à s'éduquer et se former avec le numérique. Elle se situe au croisement, d'une part, de la sociologie des usages, qui a peu développé les dimensions éducatives du numérique, et d'autre part, des sciences de l'éducation, qui ont faiblement mis en lien les usages numériques proposés aux élèves en salle de classe avec ceux développés en contexte extrascolaire. Elle s'inscrit dans la thématique des usages numériques éducatifs, telle que circonscrite par (Baron, 2014), et se veut complémentaire aux approches didactique et psychopédagogique majoritairement utilisées. Nous commençons par expliciter les fondements de cette approche. Nous présentons ensuite un aperçu de trois de ses thématiques saillantes, avant d'aborder ses implications méthodologiques et sa complémentarité avec les approches didactique et psychopédagogique, ainsi que les principaux défis qu'elle doit relever.

MOTS-CLÉS - Approche sociocritique, rapport éducatif au numérique, contexte extrascolaire.

ABSTRACT • This article aims to contribute to formalize a sociocritical approach of digital technology in education. Such a sociocritical approach focuses on the relationships between students' sociocultural profiles and contexts, and their disposition to learn with digital technology. This approach lies at the crossroads of sociology of use, on the one hand, which has granted little attention to educational uses of digital technology, and, on the other hand, education sciences, which have mainly considered educational uses of digital technology within the school context. It is part of the theme of educational uses of digital technology (Baron, 2014) and it is complementary to didactic and psychopedagogical approaches of digital technology, which are more commonly used. We first explain the foundation of this approach of digital technology in education. We then present an overview of three main research interests of this approach, before discussing its methodological implications, its complementarity with educational technology approaches and the challenges that are raised.

KEYWORDS - Sociocritical approach, educational relationship to digital technology, out-of-school context. 


\section{Introduction}

Ce texte propose une réflexion sur le domaine du numérique en éducation (aussi communément appelé « technologies de l'information et de la communication [TIC] en éducation ") en contribuant à formaliser une approche récente et montante dans le monde anglophone - que nous appelons une approche sociocritique, en empruntant le terme à (Guichon, 2011). Elle s'inscrit en réaction à certaines limites auxquelles est confronté le domaine du numérique en éducation. Engeström souligne la contradiction à laquelle font face les chercheurs en éducation : bien que la plupart d'entre eux reconnaissent que l'utilité et la validité écologique des recherches menées par le biais d'études décontextualisées aux variables contrôlées sont épistémologiquement questionnables, ils demeurent cependant victimes de pressions fortes d'ordres administratif, financier et scientifique, qui les incitent à se conformer à des principes et des approches méthodologiques issus du paradigme positiviste (Engeström, 2008) . Le domaine du numérique en éducation n'est pas exempt de ce biais en cela que les chercheurs qui y œuvrent continuent d'être sommés de prouver le bénéfice, l'intérêt et la valeur ajoutée des technologies à la fois pour légitimer des investissements financiers massifs ou pour justifier des attentes par rapport à une régénération de l'apprentissage scolaire par le biais du numérique (Eynon, 2012). Ce faisant, les recherches tendent à se focaliser sur l'impact des technologies sur les apprentissages, ce qui est assurément légitime mais non suffisant pour couvrir les différents enjeux du domaine du numérique en éducation. Comme le dit (Selwyn, 2010) :

"It is contended that more research is required that moves away from a 'means-end' way of thinking about how best to harness the presumed inherent educational potential of digital technology and, instead, focuses on the socially contested and socially shaped nature of technology », (p. 66).

Ce texte s'inscrit dans la même perspective et souhaite faire écho à celui publié en 2014 par Baron, lequel présente une synthèse pertinente de l'évolution du domaine scientifique du numérique en éducation dans l'espace francophone. Dans cet article, Baron souligne l'aspect pluridisciplinaire de ce domaine et identifie ses thématiques d'étude principales, notamment celle portant sur les «études d'usages éducatifs ». Le présent article porte sur cette dernière dimension, qui a traditionnellement été traitée par les approches didactique et psychopédagogique, et pour laquelle les apports de la sociologie sont mentionnés - voir Figure 1, p. 4 de (Baron, 2014) —, mais demeurent peu discutés. L'approche sociocritique 
du numérique en éducation que nous souhaitons formaliser dans cet article consiste, d'une part, à étudier les relations entre le profil et le contexte socioculturels des élèves et le développement d'usages numériques éducatifs, et d'autre part, à analyser les implications et les incidences sur les apprentissages, notamment scolaires, des élèves. Elle se situe au croisement de la sociologie des usages, qui a relativement peu développé les dimensions éducatives du numérique, et des sciences de l'éducation, qui ont faiblement mis en lien les usages numériques proposés aux élèves en salle de classe avec ceux développés en contexte extrascolaire. Dans la suite de ce texte, nous commençons par expliciter les fondements de cette approche. Nous esquissons ensuite les contours de son champ d'étude au moyen de trois questions vives qui lui sont liées, avant d'aborder ses implications méthodologiques et sa complémentarité avec les approches didactique et psychopédagogique, ainsi que les principaux défis auxquels les chercheurs sont confrontés.

\section{Fondements de l'approche sociocritique du numérique en éducation}

Pour présenter les fondements de l'approche sociocritique telle que nous la concevons, nous proposons dans un premier temps d'aborder ses principales prémisses. Nous discutons ensuite des usages numériques éducatifs, qui constituent une entrée d'analyse privilégiée de cette approche. Nous terminons en évoquant la posture critique qui lui est constitutive et qui la différencie des postures déterministe et instrumentaliste. Ces fondements contribuent ainsi à dresser un premier portrait d'une approche sociocritique du numérique en éducation, que nous approfondissons dans les sections ultérieures

\subsection{Quelques prémisses de départ}

L'approche sociocritique que nous formalisons dans cet article repose sur la prémisse principale que les élèves développent un rapport premier au numérique en contexte extrascolaire étant donné l'omniprésence des outils numériques dans les environnements familiaux et sociaux des pays développés (Fluckiger, 2008). Il est maintenant bien établi que les élèves n'arrivent pas en salle de classe démunis de toutes représentations et pratiques vis-à-vis de l'école, de l'apprentissage, du fonctionnement de la classe, des disciplines scolaires, etc. Par exemple, de nombreux travaux à commencer par ceux de (Penloup, 1999) - montrent que les élèves disposent d'un rapport initial à l'écriture avant que cette dernière ne fasse l'objet d'apprentissages en contexte scolaire. Ainsi, (Barré-de-Miniac, 
2000) définit le rapport à l'écriture comme l'ensemble « des conceptions, des opinions, des attitudes, de plus ou moins grande distance, de plus ou moins grande implication, mais aussi des valeurs et des sentiments attachés à l'écriture, à son apprentissage et à ses usages » (p. 13). Le "rapport à » est forgé par chaque élève sur la base de ses expériences singulières (p. ex. essais, échecs, succès, intérêt personnel) et collectives (appartenance à des groupes sociaux et culturels variés) dans un entrelacement complexe : « les groupes sociaux et culturels dans lesquels est inséré le sujet développent des usages de l'écrit et des valeurs associées à ces usages qui contribuent à la formation du rapport à l'écriture de chacun des individus, et interagissent avec les variables envisagées [...] sous l'angle de la singularité » (Barré-de-Miniac, 2000, p. 14). Au même titre que le rapport à l'écriture, nous posons que les élèves ont des représentations, des accès, des usages et des compétences numériques préalables puis concomitants à l'utilisation du numérique en salle de classe.

Ce rapport au numérique, construit en contexte extrascolaire, nous conduit à examiner deux autres prémisses. Premièrement, le contexte extrascolaire reste, tout au long de la scolarité des élèves, le principal contexte dans lequel ils construisent leur rapport au numérique. À ce titre, plusieurs études démontrent que l'accès et les usages numériques des élèves ont lieu beaucoup plus fréquemment en dehors de l'école qu'à l'école (Buckingham, 2007), (Eynon, 2008), (Alluin, 2010), (OCDE, 2010), cette dernière peinant à intégrer le numérique (Cuban, 1986), (Leask, 2011), (Maddux et Johnson, 2012), (OCDE, 2011), (Underwood et Dillon, 2011). L'écart notable entre les contextes extrascolaire et scolaire amène certains auteurs à parler d'une nouvelle fracture numérique ( new digital divide ", Buckingham, 2007, p. 112). Le contexte extrascolaire est donc doublement constitutif de la construction du rapport des élèves au numérique puisque : 1 ) une grande majorité des élèves commencent à développer leur rapport au numérique préalablement à l'école, bien qu'à des degrés variables, avec les outils accessibles dans leur environnement familial et social ; 2) le contexte extrascolaire reste, pour la suite de leur cheminement scolaire, le principal contexte d'accès et d'usages numériques des élèves. Dans cette perspective, nous avançons que le contexte scolaire, étant donné son intégration limitée du numérique à l'heure actuelle, est secondaire dans la construction du rapport des élèves au numérique, ce qui nécessite de prendre en compte finement comment les usages numériques éducatifs se construisent hors de l'institution scolaire. Les résultats de l'étude rapportée par (De Haan, 2004) vont dans ce sens. Trois hypo- 
thèses concurrentes ont été testées pour expliquer les variations de compétences numériques entre élèves : l'instruction hypothesis, qui postule que les variations de compétences numériques sont dues aux différences d'équipement informatique des écoles et à la qualité de l'enseignement des compétences numériques; la selection hypothesis, qui stipule que les variations de compétences numériques proviennent des compétences intellectuelles générales des élèves; la social background hypothesis, qui pose que les compétences numériques varient essentiellement en fonction du profil et du contexte socioculturel des élèves. Les résultats de l'étude indiquent que seule la variable de l'origine sociale permet d'expliquer de manière significative les variations de compétences numériques entre élèves, ce qui invite à penser que le contexte extrascolaire, notamment le contexte familial, joue un rôle de premier plan dans le développement d'un rapport éducatif au numérique chez les élèves.

La troisième et dernière prémisse suppose que le rapport premier au numérique, construit en contexte extrascolaire, peut avoir des incidences sur les apprentissages scolaires des élèves et sur la suite de leur parcours socioprofessionnel. Bien que cette relation de cause à effet reste à préciser, plusieurs études ont déjà établi des liens entre les usages numériques variables des élèves et leurs capacités à en tirer profit à des fins notamment éducatives, voir par exemple (Hargittai, 2010), (Livingstone et Helsper, 2007). Une étude de l'OCDE (2010) sur les données du Program for International Student Assessment (PISA) 2006 conclut d'ailleurs que les performances scolaires des élèves observés sont corrélées plus fortement à leurs usages numériques en contexte extrascolaire qu'à leurs usages en contexte scolaire. Il est donc possible de penser que le rapport que les élèves ont développé vis-à-vis du numérique en contexte extrascolaire est plus ou moins riche en potentiel pour l'apprentissage et qu'il influe sur leur disposition à s'éduquer et se former avec le numérique, notamment lorsqu'il s'agit d'apprentissages scolaires.

Pour résumer, l'approche sociocritique du numérique en éducation, telle que nous la concevons, est fondée sur trois prémisses principales, que nous reformulons ici : 1 ) les élèves disposent d'un rapport au numérique, élaboré à la fois par leurs expériences individuelles et leurs appartenances à des groupes socioculturels;2) ce rapport au numérique se développe principalement en contexte extrascolaire, à la fois avant leur scolarisation et durant leur scolarité ; 3) le rapport qu'ils construisent au numérique est susceptible d'influencer leur disposition à s'éduquer et se former avec le numérique, notamment en contexte scolaire. Parce qu'elle 
concerne l'éducation, cette approche s'intéresse tout particulièrement au rapport éducatif que les élèves développent, à des degrés variables, envers le numérique, c'est-à-dire, à leur capacité à tirer profit du numérique pour s'éduquer et se former. Sur la base de ces prémisses, les tenants d'une approche sociocritique postulent que le rapport éducatif des élèves au numérique ne peut être pleinement compris qu'à la condition de prendre en compte leur contexte et leur profil socioculturels. (Selwyn, 2010) est explicite sur ce point en affirmant que "whilst perhaps not immediately apparent to the observer of a classroom setting, it would be foolhardy to attempt to explain any aspect of education and digital technology in the 21st century without some recourse to these wider influences [the social 'milieu' of technology use]» (p. 67-68). Cette insistance à prendre en compte le milieu socioculturel n'est d'ailleurs pas spécifique à l'éducation, comme le rappelle (Feenberg, 2014) : « depuis une vingtaine d'années, les recherches historiques et sociologiques critiques consacrées à la technique ont montré toute l'importance de son ancrage socioculturel » (p. 191). En ce sens, bien qu'elle se base sur des écrits principalement issus du domaine de l'éducation, les travaux antérieurs sur la technique doivent également être pris en compte pour enrichir une approche sociocritique. Terminons en précisant que le présent article se concentre sur la population des élèves, laquelle a été de loin la plus étudiée par cette approche. Il est toutefois important de reconnaitre que le rapport éducatif des acteurs scolaires (à commencer par les enseignants et les parents d'élèves) en fait partie intégrante, bien qu'il demeure peu étudié.

\subsection{Les usages numériques éducatifs comme point d'entrée de l'approche sociocritique}

L'étude des usages numériques éducatifs constitue un point d'entrée de premier choix dans l'optique d'une approche sociocritique. Les travaux sur la cognition distribuée (Hutchins, 1995), (Hutchins, 2000) offrent un arrière-plan théorique pertinent pour aborder la dimension éducative que peuvent revêtir les usages numériques des élèves, tant dans leur quotidien scolaire qu'extrascolaire. En relevant la part de l'environnement humain («versant social ») et de l'environnement matériel («versant écologique ») dans le fonctionnement cognitif des individus (Conein, 2004, p. 57), la cognition distribuée permet d'envisager le numérique comme un ensemble d'artefacts cognitifs (Norman, 1998), c'est-à-dire :

«comme partenaires dans l'activité cpu"mö evkxkves og ni t ifs (Nor rom l'utilise. Ils peuvent ainsi être considérés comme des ressources permettant d'alléger 
les tâches cognitives d'attention, de raisonnement, de mémorisation, de planification, etc., chez l'usager dans la mesure où ils prennent en charge une partie de l'activité cognitive humaine ». (Millerand, 2002, p. 194).

Bien que la théorie de la cognition distribuée n'ait pas été élaborée spécifiquement pour les situations d'apprentissage avec le numérique, plusieurs auteurs ont déjà opéré des prolongements en lien avec l'apprentissage collaboratif à distance, p. ex. (Henri et Lundgren-Cayrol, 2001) et les environnements personnels d'apprentissage (Charlier, 2014). Appliqué aux usages numériques des élèves, le numérique peut être entendu comme un ensemble d'artefacts cognitifs susceptibles, à des degrés divers, de soutenir les apprentissages des élèves, en même temps que de modifier leur manière d'apprendre.

Parce qu'ils peuvent utilement s'appliquer à l'apprentissage au-delà des murs de la salle de classe, la cognition distribuée et le concept d'artefact cognitif permettent d'étudier les usages numériques éducatifs dans une pluralité de formes allant du formel à l'informel et dans une pluralité de contextes. Il existe plusieurs typologies sur les types d'apprentissage formel-informel (Brougère et Bezille, 2007). La majorité d'entre elles, par exemple (Coombs et Amhed, 1974), (Livingstone, 2001), (Mocker et Spear, 1982), caractérisent les types d'apprentissage à partir des situations dans lesquelles ils ont émergé. À titre d'exemple, le contexte institutionnel est majoritairement envisagé en lien avec l'apprentissage formel alors que le contexte extrascolaire serait davantage porteur d'apprentissage informel ou autre. Cette distinction des usages numériques éducatifs en fonction des contextes connaît toutefois des limites, comme le démontrent (Furlong et Davies, 2012) et (Fluckiger, 2011), en relevant que le contexte extrascolaire peut comporter des types d'apprentissage formel, par exemple lorsque les élèves utilisent le numérique à domicile pour compléter un travail scolaire. Aussi, plutôt que de «forcer » une catégorisation des usages numériques éducatifs en fonction des contextes, il nous semble plus adéquat de reconnaître que le numérique offre aux jeunes des possibilités d'apprentissage qui brouillent les frontières entre les apprentissages formels-informels (UNESCO, 2011) et qui tendent à faire tomber les lisières institutionnelles classiques entre la maison, l'école et les loisirs (Furlong et Davis, 2012), (Lai et al., 2013). Dans cette perspective, les usages numériques éducatifs gagnent donc à être appréhendés dans le cadre de parcours d'usages numériques éducatifs allant du formel à l'informel, en saisissant leur dynamique dans le temps et dans l'espace et en 
considérant les enchâssements entre les réalités en ligne et hors-ligne des élèves (Collin, 2014), (Guichon, 2015).

\subsection{Une posture critique}

L'approche sociocritique que nous adoptons, parce qu'elle adresse de front les dimensions socioculturelles qui contribuent à façonner le rapport des élèves au numérique, implique nécessairement une posture critique vis-à-vis de la technique. Puisque les perspectives critiques de la technique sont variées (George, 2014), il importe de situer celle que nous adoptons, de préciser qu'elle s'inscrit dans la lignée des travaux de l'école de Francfort et résonne avec ceux de Feenberg, en postulant que «all knowledge, even the most scientific or "commonsensical," is historical and broadly political in nature " (Friesen, 2008). Le projet de Feenberg (Feenberg, 2004), (Feenberg, 2005), (Feenberg, 2014) consiste à réinscrire la part de l'homme et de son expérience dans la technique. Pour ce faire, il appréhende cette dernière non plus selon le seul principe de rationalité technique relevant essentiellement d'acteurs dominants (les " technocrates"), mais comme la conséquence de valeurs et de choix sociaux, politiques et économiques auxquels les sociétés devraient pouvoir participer, notamment pour assurer leur bien-être. Ce faisant, la technique devient un « terrain de lutte entre des types d'acteurs entretenant des relations différentes à la technique et au sens» (Feenberg, 2004, p. 17). Ceci est bien illustré par cet auteur lorsqu'il prend l'exemple d'Internet, qui est à la fois objet de contrôle de certains groupes d'intérêt et creuset d'initiatives et de résistance pour d'autres (Feenberg, 2014). De manière globale, la perspective critique que nous adoptons a pour finalité de mettre au jour les rapports de force, les jeux de pouvoir et les intérêts multiples et contradictoires, qu'ils soient de nature politique, économique, sociale ou autre, qui façonnent l'objet d'étude et les savoirs qui lui sont relatifs (Bayne, 2014), (Buckingham, 2007), (Friesen, 2008), (Friesen, 2013), (Warschauer, 1998). Cela ne conduit pas nécessairement à dénoncer les intérêts de certains aux dépens d'autres, mais à partir de cet état de fait pour élaborer des savoirs complémentaires ou alternatifs, qui tiennent compte des enjeux à l'œuvre, et qui, ultimement, visent à contribuer à davantage de justice sociale. Ainsi, pour (Feenberg, 2005), la tendance technocratique qui domine actuellement la technique entraîne certains bénéfices et en écarte d'autres qui pourraient pourtant occasionner des retombées durables et positives sur le plan social. 
La posture critique ainsi envisagée se démarque de deux autres postures : la posture déterministe, aussi appelée « essentialiste " par (Hamilton et Friesen, 2013) et la posture instrumentaliste, telle qu'identifiée par (Warschauer, 1998). La première part du principe que le numérique dispose de propriétés éducatives inhérentes qui sont à même de soutenir l'enseignement et l'apprentissage, ce qui a pour conséquence néfaste de surestimer le rôle du numérique et de sous-estimer le rôle des acteurs et des contextes d'enseignement et d'apprentissage (Collin et Karsenti, 2012a). À l'opposé, la posture instrumentaliste envisage le numérique comme un ensemble d'outils prêts à être utilisés pour servir les fins des utilisateurs selon une perspective où les technologies sont perçues comme neutres et ne comportant pas de valeurs propres (Feenberg, 1991, p. 5). Cette deuxième posture pêche par l'excès inverse de la posture déterministe, en sous-estimant les influences différenciées des outils numériques spécifiques sur les individus et les groupes dans leurs pratiques sociales et culturelles (Millerand, 2003). Appliquée au numérique en éducation, la posture instrumentaliste ne parvient pas à rendre compte de la manière dont le numérique affecte le rapport éducatif des élèves au numérique (Warschauer, 1998). Dans un cas comme dans l'autre, ces deux postures tendent à décontextualiser le rapport éducatif au numérique, comme si ce dernier se développait de façon autonome, indépendamment de la singularité, du profil et du contexte socioculturels propres à chaque élève. Nous rejoignons alors (Bayne, 2014) pour dire que :

"such over-simplification does the field no favours, either as a domain of research or as a domain of practice. Casting the technological and the social as isolated from each other in the context of digital (and post-digital) education merely robs the field of its complexity and richness, reducing our capacity to understand it as a domain of genuine social significance » (p. 5-6).

Par ailleurs, la posture critique présente aussi l'intérêt de mettre au jour les idéologies qui opèrent dans le domaine du numérique en éducation, tant chez les administrateurs politiques, que les praticiens et les chercheurs. Friesen définit une idéologie comme « a set of ideas or a kind of knowledge that is used to justify actions of social and political consequence and that is considered so obviously commonsensical or natural that it is placed beyond criticism » (Friesen, 2008). Avec cette définition en tête, il est possible de constater qu'une partie des développements numériques en éducation est accompagnée de discours idéologiques qui véhiculent des attentes souvent disproportionnées chez les acteurs politiques, professionnels et scientifiques (George, 2014), (Gouseti, 2010), (Selwyn, 2010). (Feenberg, 
2001) pointe bien la forte charge idéologique du numérique en éducation en soulignant que le fait que le numérique fonctionne ou ne fonctionne pas est finalement moins important que la place que cela occupe dans l'imaginaire d'un certain nombre de réformistes de l'éducation.

Pour prendre un exemple récent, citons le cas des Massive Open Online Courses (MOOC, cours en ligne ouverts et massivement distribués), que Coursera présente par le biais d'un affichage humaniste : «nous imaginons un futur où tout le monde aura accès à une éducation de première qualité » (Coursera, 2014). Pourtant, plusieurs études, p. ex. (Christensen et al., 2013) démontrent que le profil d'étudiants bénéficiant des MOOC est majoritairement déjà éduqué, ce que (Laurillard, 2014) résume de façon cynique ainsi : «the problem MOOCs succeed in solving is: to provide free university teaching for highly qualified professionals » (en ligne). Ainsi, si les MOOC présentent un certain potentiel pédagogique, une partie des discours sur les MOOC sont idéologiques et passe notamment sous silence les intérêts commerciaux de Coursera (Kolowich, 2013) et des enjeux éducatifs tels que la déqualification possible du corps professoral (Feenberg, 2001). Face à ce type de discours dont les MOOC représentent la dernière manifestation en date, une posture critique permet d'une part de mettre au jour les idéologies, les intérêts et les rapports de force à l'œuvre, et d'autre part, d'apprécier les apports de certains dispositifs, applications ou outils numériques en tenant compte de la singularité des multiples contextes et profils socioculturels des étudiants à qui ils s'adressent, ce qui représente, à notre sens, un gage de succès pour une meilleure actualisation de leur potentiel.

\section{Aperçu de trois thématiques saillantes de l'approche sociocritique}

Dans cette section, nous esquissons les contours du champ d'étude d'une approche sociocritique à travers trois thématiques saillantes, suivant un découpage sans doute discutable mais qui illustre bien les enjeux de recherche de cette approche. Nous ne prétendons pas présenter une revue de la littérature exhaustive de chacune d'entre elles mais davantage donner un aperçu de la manière dont l'approche sociocritique que nous formalisons dans cet article s'est structurée et des principaux points qu'il reste à approfondir. 


\subsection{Congruence des contextes scolaire et extrascolaire dans la construction du rapport éducatif des élèves au numérique}

L'inclusion du profil et du contexte socioculturels des élèves dans l'étude de leur rapport éducatif au numérique pose d'emblée la question de la congruence et des transferts possibles entre les contextes scolaire et extrascolaire. Les études empiriques et conceptuelles consultées permettent de dégager quelques tendances à cet égard. En premier lieu, l'étude de (Fluckiger, 2011) établit que les outils numériques personnels (p. ex. réseaux sociaux, téléphones cellulaires, courriels personnels) peuvent être mis à profit pour des usages numériques éducatifs formels dans le cadre d'un travail d'équipe entre étudiants universitaires, alors que les outils numériques institutionnels (p. ex. courriels institutionnels, forums de discussion disponibles dans les environnements numériques d'apprentissage) sont délaissés. Ainsi, le contexte extrascolaire peut accueillir une variété d'usages numériques éducatifs, allant du plus formel au plus informel (voir section 2.2.) et cumulés à des usages numériques non éducatifs. En revanche, le contexte scolaire semble davantage privilégier les usages numériques éducatifs formels aux dépens d'autres types d'usages numériques éducatifs (Buckingham, 2007), (Male et Burden, 2013).

Cet écart entre, d'une part, la diversité des types d'usages numériques éducatifs en contexte extrascolaire et, d'autre part, la quasi-exclusivité des usages numériques éducatifs formels en contexte scolaire semble relever plus largement d'une divergence d'autonomie et d'initiative dont disposent les élèves (Buckingham, 2007). Plus précisément, le degré d'autonomie et d'initiative des élèves en contexte extrascolaire, conséquent de l'évolution sociale du statut d'adolescent dans les sociétés occidentales (Schneider, 2013), leur permettrait de développer de nouveaux styles d'apprentissage avec le numérique (p. ex. développement de compétences par essai-erreur, collaborations ponctuelles sur demande, exploration et expérimentation non linéaires, etc.), que les contraintes scolaires ne permettraient pas aisément de réinvestir en salle de classe. Comme le souligne (Fluckiger, 2008) :

«Les TIC [utilisées en contexte extrascolaire] sont perçues comme émancipatrices par les jeunes qui peuvent accéder à des contenus numériques sans passer par les médiations parentales habituelles, ou entretenir grâce aux outils de communication numérique une sociabilité de pairs au sein même du domicile familial. Cette dimension émancipatrice est ab- 
sente des usages scolaires, qui sont nécessairement sous contrainte » (p. $53)$.

De leur analyse d'études empiriques, (Bourque et al., 2013) concluent que les activités pédagogiques recourant à la Toile sont rarement en mesure de développer la pensée critique des élèves en contexte scolaire, car "l'apprenant, soumis à un contrôle interne et externe, conscient de la pression sociale normative et de l'autorité du maître, dispose de bien peu de latitude » (p. 15). On retrouve ici la faible autonomie que l'école permet aux élèves, d'après (Furlong et Davies, 2012). Cette divergence de fond entre les contextes scolaire et extrascolaire placerait les élèves dans une situation de tension lorsque que l'école leur impose, en contexte scolaire, des outils numériques associés au contexte extrascolaire, tout en délégitimant le degré d'autonomie et d'initiative qui les accompagne habituellement (Clark et al., 2009). Cette divergence d'autonomie et d'initiative contribuerait à expliquer pourquoi l'intégration du numérique en salle de classe a souvent donné des résultats mitigés sur les apprentissages scolaires (Ito et al., 2013), (Male et Burden, 2013).

Dans la perspective de cette thématique, un des principaux enjeux actuels consiste à mieux définir et catégoriser les types d'usages numériques qu'inclut le rapport éducatif au numérique, notamment en intégrant ceux qui ont lieu en contexte extrascolaire (voir section 2.2.).

\subsection{Inégalités numériques en éducation}

La thématique des inégalités numériques en éducation s'intéresse aux relations entre le rapport des élèves au numérique, les déterminants de ce rapport et la capacité des élèves à en tirer profit dans une visée éducative. Sur le plan empirique, il s'agit donc, dans un premier temps, de mettre au jour les variations du rapport des élèves au numérique et les facteurs associés (p. ex. sexe, revenu des parents, nombre d'outils numériques accessibles, types et nombre d'usages d'Internet, etc.), et dans un second temps, d'étudier les incidences sur le développement d'un rapport éducatif au numérique.

Le premier point a été le plus étudié, de sorte que nous n'y reviendrons pas ici, voir p. ex. (Collin et Karsenti, 2013). Nous nous contenterons simplement de rappeler que la qualité du rapport des élèves à Internet semble prendre la forme d'une «échelle d'opportunités » («ladder of opportunities »), (Livingstone et Helsper, 2007), (Wei, 2012), présentant une cumulation progressive allant de quelques usages basiques au 
départ, et partagés par une majorité d'élèves (par exemple, jouer à des jeux en ligne), à l'ajout d'usages plus complexes et moins répandus, qui impliquent notamment la participation (p. ex. partages de fichiers) et la création (p. ex. montages vidéo, programmation). Aussi, l'échelle d'opportunités invite à penser que certains usages numériques éducatifs se " greffent » éventuellement à des usages non éducatifs préexistants (Livingstone et Helsper, 2007). Il est important de noter que les élèves ne progressent pas également à travers l'échelle d'opportunités : certains d'entre eux peuvent demeurer à un niveau de base, alors que d'autres accèdent à des niveaux intermédiaires ou élevés. En somme, le numérique ouvre des opportunités éducatives plus ou moins riches, que les élèves saisissent à des degrés divers, notamment suivant leur profil et leur contexte socioculturels. Les variables déterminant le positionnement de chaque élève sur l'échelle d'opportunités peuvent être nombreuses et combinées les unes aux autres. Parmi les plus récurrentes dans la littérature scientifique, notons l'âge mais également le niveau socioéconomique des élèves et de leurs parents, par exemple (Gire et Granjon, 2012), (Wei, 2012), ce qui ancre résolument les inégalités numériques dans des inégalités sociales plus larges, bien que leurs relations ne soient pas toujours symétriques (Granjon, 2009). En France, l'ouvrage dirigé par Langouët (Langouët, 2000) constitue un des premiers portraits approfondis des inégalités numériques appliquées aux jeunes et avec des implications éducatives (voir notamment les textes de Baron, Linard et Porcher).

Les incidences des inégalités numériques sur le développement d’un rapport éducatif au numérique ont été beaucoup moins explorées et les résultats actuels sont parfois contradictoires. Ainsi, (Fairlie et Robinson, 2013) ont mené une étude quasi-expérimentale sur les élèves ne possédant pas personnellement d'ordinateurs. Une partie d'entre eux (559 élèves sur 1123) ont été équipés d'un ordinateur à domicile. Il en ressort que les performances scolaires entre les élèves ayant été dotés d'un ordinateur et ceux n'ayant toujours pas d'ordinateur n'ont fait état d'aucune différence significative entre les élèves. Plusieurs limites invitent toutefois à prendre ces résultats avec prudence : 1) le temps de traitement était relativement court (les élèves ont reçu les ordinateurs à la fin de l'automne et étaient évalués d'après les examens ministériels en fin d'année scolaire) pour espérer noter des changements substantiels dans la performance scolaire ; 2) les élèves et leurs parents n'ont pas reçu de formation particulière dans l'utilisation de l'ordinateur, alors que les premiers étaient relativement jeunes (6 à 10 ans). Sachant que les enjeux 
éducatifs du numérique se situent davantage au niveau des usages que de l'accès (Hargittai, 2002) ${ }^{1}$, fournir un ordinateur à chaque élève sans se préoccuper des usages qui en sont faits présente peu de chance, en soi, d'aboutir à des résultats concluants sur le plan éducatif ; 3) enfin, les auteurs n'ont pas pu isoler la cause de l'absence d'ordinateur à la maison, pour chacun des foyers concerné. Or l'absence d'ordinateur ne constitue pas de facto une manifestation des inégalités numériques, comme le reconnaissent d'ailleurs les auteurs (p. 2-3).

L'étude multi-cas d'Angus, Snyder et Sutherland-Smith (Angus et al., 2004) auprès de quatre familles défavorisées d'élèves montre bien comment le capital culturel de chaque famille oriente, chez les élèves, le développement d'un rapport plus ou moins éducatif au numérique en contexte extrascolaire. L'étude de (Rafalow, 2014) envisage une autre incidence des inégalités numériques en éducation, en s'intéressant aux relations entre le niveau socioéconomique des écoles, les usages du tableau blanc interactif (TBI), les représentations enseignantes et la culture institutionnelle concernant la valeur éducative du numérique. Cette étude multi-cas portait sur trois enseignants dans des milieux volontairement contrastés (niveaux socioéconomiques élevé, moyen et faible) et s'appuyait aussi sur des entrevues avec leurs collègues, afin de dresser un portrait de la «culture » de l'école. Rafalow conclut que des différences notables se font jour selon le niveau socioéconomique : l'enseignant de l'école de milieu socioéconomique favorisé fait état d'usages innovants du TBI dans l'optique de continuer à offrir un enseignement de qualité aux élèves, notamment sous la pression des parents. Tout aussi dynamique dans ses usages du TBI, l'enseignant de l'école de milieu moyennement favorisé perçoit principalement le TBI dans sa dimension motivationnelle et l'utilise moins fréquemment, ce qui semble être un contrecoup du manque d'adhésion de certains collègues. Enfin, l'enseignant de l'école de milieu défavorisé utilise principalement le TBI en reproduisant les pratiques du tableau noir et sans y apposer de nouvelles valeurs éducatives. Bien que ces résultats ne soient pas généralisables, ils laissent penser que les représentations des enseignants et la culture de chaque école sont influencées par le niveau socioéconomique du milieu concerné, et qu'elles contribuent à déterminer le potentiel attribué au numérique et les usages qui en sont faits. L'étude quantitative de (Nunn et al., 2002) va dans le même sens. Elle conclut, à partir de différents indicateurs appliqués aux écoles publiques du Maryland (États-Unis), que les enseignants des écoles de milieu défavorisé utilisent moins fréquemment et moins efficacement 
le numérique, complémentairement aux désavantages numériques observés chez les élèves de ces écoles.

Ces résultats semblent indiquer que les inégalités numériques présentent des incidences tant sur le plan des usages numériques éducatifs des élèves que sur celui des pratiques techno-pédagogiques des enseignants.

\subsection{Rapport éducatif des élèves migrants ou issus des minorités ethnoculturelles au numérique pour leur intégration linguistique et scolaire}

Une troisième thématique, sans doute la moins travaillée à l'heure actuelle, concerne le rapport éducatif des élèves migrants ou issus des minorités ethnoculturelles. L'origine de cette thématique se trouve dans le constat que le rapport au numérique n'est pas neutre et qu'il varie suivant les traits culturels propres à chaque groupe (Dibakana, 2010), (Erumban et De Jong, 2006), (Nistor et al., 2014), bien qu'il s'agisse d'aspects « plus subjectifs, difficilement quantifiables, et qui conservent pourtant toute leur importance dans l'analyse des comportements d'adoption [du numérique par les individus] " (Kharbeche, 2006, p. 90). Ces traits culturels sont susceptibles d'influencer le rapport des individus au numérique, suivant un processus de «resignification» (Mattelart, 2009). À titre d'exemple, (Agboton, 2006) observe que la tradition orale valorisée dans certaines communautés africaines, notamment comme moyen privilégié de transmettre les savoirs, peut représenter un frein pour l'adoption d'Internet, où le savoir est exposé de manière écrite dans la plupart des cas, et sans interlocuteur physique. (Daoudi, 2011) met notamment en exergue la ruralité, la vie en collectivité et la rigidité des liens hiérarchiques comme des caractéristiques culturelles supplémentaires pouvant avoir une influence sur les usagers africains.

Sur la base de ces constats, (Daoudi, 2011) invite à développer « une intégration durable, adaptée au contexte local et favorisant la pleine participation des enseignants et des élèves » (p. 114). Cette dimension culturelle a été relativement peu prise en compte dans l'étude du numérique en éducation, ce qui s'explique sans doute par le fait que la majorité des outils numériques ont été historiquement créés par des entreprises occidentales et étudiés dans des contextes scolaires occidentaux, notamment aux États-Unis (Helsper et Eynon, 2010), (Guichon, 2015).

À ces dimensions culturelles s'ajoutent des dimensions migratoires, notamment liées à l'intégration linguistique, psychologique et sociocultu- 
relle des élèves et de leurs familles (Chen et Choi Siu Kay, 2011), ainsi qu'au maintien des contacts avec leur société d'origine (Gallant et Friche, 2012). En termes proprement éducatifs, l'intégration linguistique semble avoir été l'aspect le plus étudié. À titre d'exemple, quelques études se sont penchées sur le numérique comme soutien à l'intégration linguistique des migrants, soit la manière dont ces derniers tirent ou non profit du numérique, dans des contextes d'apprentissage plus ou moins liés à l'institution éducative, pour apprendre la langue de la société d'accueil, par exemple (Collin et Karsenti, 2012b), (Kluzer et al., 2009), (Spotti et al., 2010). En revanche, ces études ont principalement porté sur les adultes et n'abordent pas l'intégration scolaire des élèves migrants.

Le rapport éducatif au numérique des élèves migrants et issus des minorités ethnoculturelles reste donc en grande partie à explorer. Il s'agit d'un double défi : d'une part, arriver à isoler les variables culturelles et migratoires des variables sociales, ce qui est complexe dans la mesure où elles sont étroitement imbriquées (Mesch et Talmud, 2011), (Schradie, 2012) ; d'autre part, parvenir à rendre compte avec acuité des traits culturels spécifiques à chaque groupe ethnoculturel et migratoire, sans tomber dans du "particularisme " empêchant toute discussion des résultats au delà des terrains étudiés.

\section{Considérations méthodologiques}

Bien que l'approche sociocritique que nous adoptons ne se prévale pas d'une méthodologie spécifique, elle tend à être davantage compatible avec certaines méthodes qu'avec d'autres. En s'intéressant aux usages numériques éducatifs des élèves tels qu'ils les pratiquent dans leur quotidien scolaire et extrascolaire, cette approche implique d'emblée des recherches descriptives plutôt qu'interventionnistes. Autrement dit, l'objectif est de documenter les pratiques numériques telles qu'elles sont, et non pas telles qu'elles devraient être (Selwyn, 2010), ce qui rend les approches de type quasi-expérimental peu adaptées (Engeström, 2008). Dans une visée descriptive, deux niveaux de collecte et d'analyse complémentaires gagnent à être considérés: les observations, c'est-à-dire les pratiques numériques effectives des élèves, et les représentations, qui renvoient à la manière dont les élèves font sens de leurs pratiques du numérique. Ces deux niveaux d'analyse sont à la fois autonomes et complémentaires l'un de l'autre : ils peuvent apparaître conjointement ou non dans un même devis méthodologique, suivant l'objectif poursuivi. Concernant les méthodes en tant que telles, l'entrevue et le questionnaire sont tout à fait adéquats 
mais présentent l'inconvénient de ne pas permettre l'observation directe des pratiques numériques éducatives, ce qui a été reproché au domaine du numérique en éducation (Ungerleider, 2002). En effet, un des enjeux méthodologiques est précisément d'accéder aux pratiques numériques effectives et dynamiques des élèves. Certaines avancées technologiques permettent cependant de repousser les limites méthodologiques qui ont longtemps grevé l'observation des pratiques numériques éducatives. Le développement du big data et du data mining (Baker et Yacef, 2009), par exemple, ouvrent des avenues très intéressantes pour observer en continu dans le temps et dans l'espace toutes les activités numériques des élèves sur un outil donné, et ce de façon systématique, automatique et discrète, ce qui n'était pas possible jusque-là. En revanche, ce type d'approche méthodologique ne permet pas d'accéder aux représentations des élèves. L'approche ethnographique présente alors une entrée de choix dans la mesure où elle conjugue à la fois la collecte d'observations et de représentations in situ (Schneider, 2013). Elle permet ainsi de documenter les pratiques numériques dans leur «écologie médiatique » (Ito et al., 2010). La recherche récente de (Boyd, 2014) sur les usages numériques des jeunes constitue un exemple de la pertinence de l'ethnographie. Cette chercheure a pu identifier, grâce à une enquête ethnographique approfondie, les principaux enjeux que génèrent les usages numériques des jeunes. En revanche, l'inconvénient de cette méthode est d'être plus intrusive pour les participants et plus chronophage pour le chercheur que les autres méthodes de collecte de données, et partielle par rapport au big data et au data mining dans les usages numériques recueillis. En somme, et sans surprise, aucune méthode n'est suffisante pour prétendre rendre compte a elle seule du rapport éducatif des élèves au numérique, dans la mesure où ce rapport est complexe et évolutif. L'enjeu pour les chercheurs s'inscrivant dans une approche sociocritique est donc de conjuguer les méthodes pour combler les lacunes des unes par les forces des autres, selon les principes de l'approche mixte (Teddlie et Tashakkori, 2009), (Creswell, 2014).

\section{Complémentarité des approches didactique, psychopédagogique et sociocritique dans l'étude du numérique en éducation}

Après avoir examiné les enjeux méthodologiques d'une approche sociocritique, nous proposons de situer cette dernière au sein de l'étude des usages numériques éducatifs, traditionnellement menée par les approches didactique et psychopédagogique. 
La principale distinction d'une approche sociocritique avec les approches didactique et psychopédagogique est liée à l'étendue de leurs objets d'étude respectifs. Parce que les approches didactique et psychopédagogique se concentrent habituellement sur la situation d'enseignement et d'apprentissage et ses acteurs (en premier lieu, l'enseignant et les apprenants), certains auteurs relèvent que les usages numériques éducatifs observés laissent de côté certains autres usages numériques éducatifs, notamment ceux développés à l'initiative des élèves en contexte extrascolaire. Leander, Philipps et Taylor (Leander et al., 2010) rendent compte de ce point en prenant la métaphore de la classroom as container :

"The classroom-as-container constructs not only particular ways of speaking and writing in educational research, but also systems of rules concerning how meaning is made (Foucault, 1972). This discourse functions as an imagined geography of education, constituting when and where researchers and teachers should expect learning to take place. This dominant discourse shapes educational research practice and perspectives, we posit, even when research questions cross in school and out of school borders ». (p. 329).

Cela invite à élargir la perspective avec laquelle est appréhendé le rapport éducatif des élèves au numérique en prenant en compte toute sa complexité et sa polymorphie, transversalement aux contextes scolaires et extrascolaires. C'est d'ailleurs ce que tentent d'opérer plusieurs concepts, tels que les learning lives (Erstad et Arnseth, 2013), le connected learning (Ito et al., 2010), ou encore le mobile learning (Berge et Muilenburg, 2013), qui présentent des directions fructueuses pour élargir la conception des usages numériques éducatifs tout en veillant à ne pas négliger l'apprentissage scolaire. Ces concepts sont récents de sorte que nous ne sommes pas en mesure de les discuter et de les différencier avec finesse. Retenons toutefois que leurs conceptions respectives de l'apprentissage partagent plusieurs traits communs : 1 ) ils relèvent tous la nécessité d'appréhender l'apprentissage non pas en vase-clos, mais de façon continue dans le temps (suivi longitudinal) et dans l'espace, incluant le contexte scolaire (suivi transcontextuel) ;2) ils abordent l'apprentissage dans ses dimensions cognitives mais également affectives et expérientielles ; 3) enfin, ils l'abordent dans ses «pratiques épistémiques » (Kumpulainen et SeftonGreen, 2014, p. 8) formelles, informelles et autres. À ce titre, ces concepts s'avèrent prometteurs pour penser le développement d'un rapport éducatif des élèves au numérique de manière intégrée. Ils sont, du même coup, capables de concilier l'apprentissage scolaire et les autres types d'apprentissage. Nous reconnaissons toutefois avec (Fluckiger, 2014) que 
« si l'hypothèse de continuité s'avère heuristique pour penser les pratiques académiques, elle porte le risque de minorer certaines de leurs spécificités, en omettant de mettre l'accent sur le rôle du contexte dans le formatage des usages » (p. 15).

Cette approche ouvre ainsi une perspective complémentaire et compatible avec les approches didactique et psychopédagogique. La figure 1 illustre trois dimensions d'analyse possibles des usages numériques éducatifs. La première renvoie à l'approche didactique et la seconde, à l'approche psychopédagogique. Ces deux premières dimensions d'analyse se situent en contexte scolaire. La troisième est une approche sociocritique qui positionne une troisième dimension d'analyse.

Parce qu'elle s'intéresse aux relations entre le profil et le contexte socioculturel des élèves et le développement d'un rapport éducatif au numérique, cette approche ne se limite pas à l'étude des usages numériques éducatifs en contexte extrascolaire. Elle s'intéresse également à leur congruence avec les usages numériques éducatifs proposés aux élèves en contexte scolaire. Elle englobe donc à la fois les contextes scolaires et extrascolaires. Les pointillés signifient la porosité entre les trois dimensions d'analyse des usages numériques éducatifs, alors que la flèche qui les traverse exprime leur transversalité, à la fois à travers les contextes (scolaire et extrascolaire) et les dimensions d'analyse (didactique, psychopédagogique et sociocritique).

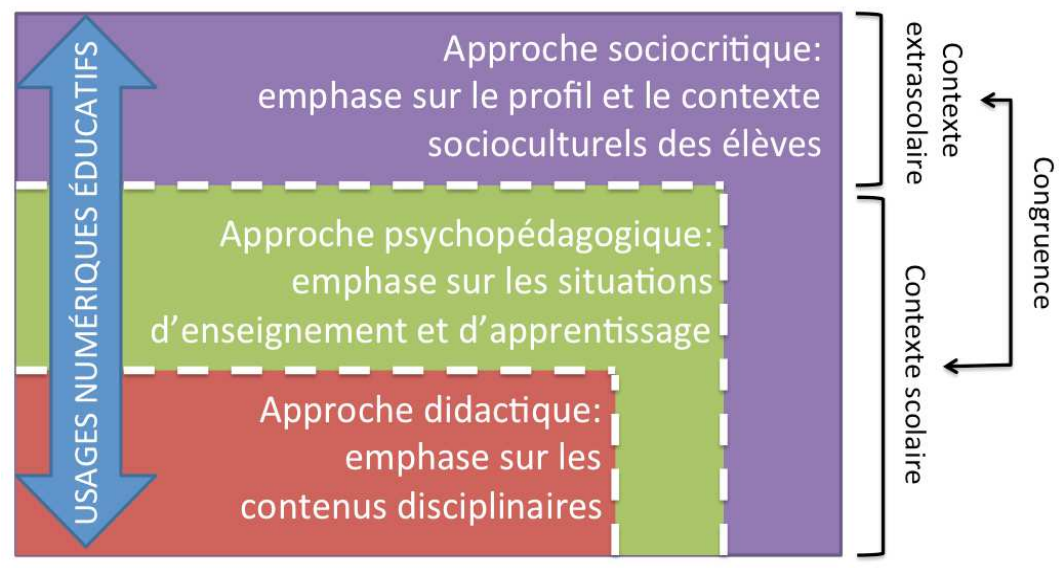

Figure 1 - Les approches didactique, psychopédagogique et sociocritique en tant que dimensions d'analyse complémentaires pour l'étude des usages numériques éducatifs 
Il ne s'agit pas de favoriser une de ces trois dimensions d'analyse aux dépens des autres, mais plutôt de reconnaitre que l'étude du rapport éducatif des élèves au numérique, en tant que domaine de recherche, ne peut être pleinement accomplie que par la mutualisation des connaissances issues de ces trois dimensions d'analyse. Nous rejoignons ainsi (Selwyn, 2010) lorsqu'il souligne qu'une approche sociocritique offre simplement une dimension supplémentaire à l'étude des technologies éducatives en complément à une perspective davantage focalisée sur les apprentissages qui a dominé le domaine au cours des vingt-cinq dernières années.

\section{Défis d'une approche sociocritique}

Comme toute approche théorique, une telle approche du numérique en éducation comporte aussi son lot de défis à relever et de glissements conceptuels à éviter. En premier lieu, il importe de reconnaître que cette approche est en émergence et qu'elle porte sur un objet (les usages numériques éducatifs) lui-même évolutif au gré des avancées technologiques et des changements socioculturels, tant à l'échelle de l'individu que de la société. Cette approche, tout comme son objet d'étude, ne sont donc pas encore stabilisés. Ainsi, ses fondements critiques, la délimitation de ses objets de recherches, la théorisation de ces concepts-clés dans un tout systémique et inclusif se doivent d'être précisés. Un des enjeux consiste donc à poursuivre ses efforts de structuration en arrimant solidement les acquis sur lesquels elle repose et les nouveaux développements conceptuels, méthodologiques et empiriques qui opèrent rapidement dans le domaine du numérique en éducation. À l'heure actuelle, les recherches empiriques se situant dans une approche sociocritique ne peuvent donc faire l'économie d'une réflexion théorique sur les apports, les limites et les implications de cette approche sur leurs travaux de recherche et sur le domaine du numérique en éducation d'une manière générale. Aussi, ce texte ne prétend pas tant présenter un aboutissement que contribuer aux réflexions et à la formalisation d'une approche sociocritique du numérique en éducation dans l'espace francophone.

En deuxième lieu, définir et catégoriser les usages numériques éducatifs reste problématique à l'heure actuelle. En s'ouvrant sur le contexte extrascolaire, cette approche complexifie considérablement les usages numériques éducatifs et le concept même d'apprentissage. À titre d'exemple, les usages numériques relationnels et ludiques ne forment pas des usages numériques éducatifs par eux-mêmes. Ils peuvent toutefois le devenir s'ils sont mis au service d'intentions ou de retombées éducatives, 
plus ou moins délibérées, lorsque des élèves utilisent Facebook pour faire un travail en équipe ou encore lorsqu'ils jouent à un jeu en ligne en anglais et qu'ils s'approprient le vocabulaire utilisé (Thorne et al., 2012). Ce faisant, les distinctions entre certains types d'apprentissages, notamment informels, et les pratiques de loisirs et de socialisation des élèves tendent à s'infléchir, ce qui soulève de nouvelles questions : jusqu'où un usage numérique est-il éducatif ? À partir de quand n'a-t-il plus de valeur éducative ? En somme, de quoi est fait un rapport "éducatif » au numérique ? Ou, pour le dire avec (Kumpulainen et Sefton-Green, 2014) :

"Efforts to understand the dynamic processes of learning situated across space and time, beyond the here and now, are presently challenging traditional definitions of learning and education. How can we conceptualize learning in a way that is able to explain the increasing complexity, connectivity, and velocity of our times?» (p. 8).

Finalement, la posture critique sous-jacente à une approche sociocritique, parce qu'elle reconnaît que les usages numériques sont ancrés dans des rapports de force et des intérêts dépassant le seul contexte scolaire, est portée à mettre au jour les déterminants structuraux qui sont en jeu, notamment dans l'optique de rendre compte des inégalités numériques en éducation. Poussé à l'extrême, ce raisonnement pourrait glisser dans l'excès inverse du déterminisme technologique, c'est-à-dire dans un déterminisme social abusif — voir (Granjon, 2004) à propos de l'École de Francfort - en présentant les usages numériques éducatifs comme relevant principalement du positionnement social des élèves. Pour montrer l'intérêt de nuancer cette position, prenons comme exemple le cas des élèves non usagers d'Internet. Dans l'optique d'un déterminisme social abusif, les élèves non connectés pourraient être compris comme la manifestation la plus poussée de l'exclusion numérique. Pourtant, (Livingstone et Helsper, 2007) identifient plusieurs profils de jeunes non connectés : les voluntary drop-outs (have access, stopped using) ; les involuntary drop-outs (lost access, stopped using) ; les potential users (have access, never used) ; les Internet excluded (no access, never used). Si les profils 2 et 4 semblent effectivement relever des inégalités numériques, les profils 1 et 3 ne permettent pas de généraliser cette situation à l'ensemble des cas de non usage. Les déterminants socioculturels restent donc pertinents à étudier mais ne sauraient constituer l'unique cadre de référence d'une approche sociocritique. 


\section{Conclusion}

Pour rappel, l'objectif du présent article était de contribuer à formaliser une approche sociocritique et son apport au domaine du numérique en éducation, notamment à la thématique des usages numériques éducatifs, telle que circonscrite par (Baron, 2014). Pour ce faire, nous avons commencé par circonscrire les fondements d'une telle approche, avant d'identifier les contours de son champ d'étude à travers trois thématiques saillantes. Nous avons également situé dans leurs grandes lignes les implications méthodologiques de cette approche, sa complémentarité avec les approches didactique et psychopédagogique et les limites auxquelles elle fait face.

Entre les discours zélotes des tenants du numérique (par exemple, le numérique comme preuve de la capacité de l'école à innover, l'intégration du numérique comme moyen privilégié de motiver les élèves) et les discours dénonçant les effets du numérique sur les apprentissages (déconcentration, culture du loisir plutôt que de l'effort, perversion des missions éducatives prioritaires), l'approche sociocritique du numérique en éducation que nous adoptons se donne comme perspective d'approfondir la compréhension du rapport plus ou moins éducatif que les élèves développent à l'égard du numérique, ainsi que les causes et les conséquences sur l'apprentissage et le cheminement scolaire des élèves. Pour poursuivre le développement de cette approche, plusieurs niveaux d'action nous semblent impératifs : 1) sur le plan théorique, plusieurs concepts brièvement évoqués dans le présent article, tels que l'échelle d'opportunités numériques, les inégalités numériques, le mobile learning, le connected learning, les learning lives, semblent prometteurs pour interpréter les résultats empiriques du domaine. En revanche, il s'agit de concepts relativement récents qui se doivent d'être précisés et mis à l'épreuve du terrain avant de servir de base théorique solide. 2) sur le plan empirique, il convient de déployer des programmes de recherche capables, à partir de méthodologies qualitatives, quantitatives, longitudinales et comparatives, de documenter finement:a) les pratiques numériques éducatives de divers profils socioculturels d'élèves (recueil d'observations); b) le sens que ces derniers leur attribuent (recueil de représentations). En outre, comme le rapport au numérique est multidimensionnel, mobile et évolutif, les usages numériques éducatifs gagneraient à être interprétés de façon holistique au sein de parcours d'usages numériques éducatifs, eux-mêmes enchâssés de façon complexe dans des activités et des réalités offline. L'enjeu consiste donc à 
savoir comment combiner au mieux des méthodes pertinentes susceptibles de capturer toute la complexité du rapport éducatif au numérique. Conjointement à l'étude du rapport éducatif des élèves au numérique, il convient de se pencher davantage sur le rapport éducatif que développent les acteurs scolaires (notamment les enseignants et les parents d'élèves), ce qui a peu été investigué jusqu'à maintenant. Or il s'agit d'aspects dont elle ne pourra pas faire l'économie si elle souhaite inscrire l'élève dans l'agencement relationnel qui est le sien au quotidien. Outre l'étude du rapport éducatif des élèves et des acteurs scolaires au numérique, cette approche ne peut se dispenser de mettre au jour des discours politiques, institutionnels, médiatiques, économiques et scientifiques afin de montrer comment ils influent sur les politiques et les pratiques d'intégration du numérique en contexte scolaire, avec quels intérêts, quels agendas et quelles conséquences. 3) Enfin, comme une telle approche du numérique en éducation est récente et encore peu structurée dans l'espace francophone, les chercheurs qui s'y intéressent gagneraient à intensifier les collaborations afin de mutualiser leurs expertises, leurs initiatives et leurs contributions à l'avancement des connaissances.

1 On gardera à l'esprit que l'accès reste, malgré tout, une condition nécessaire, mais non suffisante des usages numériques éducatifs, et qu'il reste problématique dans plusieurs pays voir p. ex. (Brown et Czerniewicz, 2010) pour le cas de l'Afrique du Sud. 



\section{BIBLIOGRAPHIE}

AGBOTON J. (2006). L'impact des réalités socioculturelles locales sur l'appropriation de l'Internet en Afrique subsaharienne. In C. Ammi (dir.), Innovations technologiques : aspects culturels et mondialisation. Paris. Lavoisier, pp. 129-148.

ALLUIN F. (2010). Les technologies de l'information et de la communication (TIC) en classe au collège et au lycée :éléments d'usages et enjeux. Paris: Ministère de l'Éducation nationale, de l'enseignement supérieur et de la recherche, Direction de l'évaluation et de la prospective, 2010, 85 p. Disponible sur internet : http://media.education.gouv.fr/file/197/18/9/Dossier197 158189.pdf (consulté le 30 juin 2014).

ANGUS L., SUTHERLAND-SMITH W., SNYDER I. (2004). ICTs and educational (dis)advantage: cultural resources and the digital divide. In Walford, G. et Jeffrey, R. A. (dir), Ethnographies of educational and cultural conflicts: strategies and resolutions. Amsterdam. Elsevier JAI, pp. 45-66.

BAKER R., YACEF K. (2009). The State of Educational Data Mining in 2009: A Review and Future Visions. Journal of Educational Data Mining, Vol. 1, n 1, p. 3-17.

BARON G.-L. (2014). Élèves, apprentissages et " numérique »: regard rétrospectif et perspectives. Recherches en Éducation, Vol. 18, $\mathrm{n}^{\circ}$ 2, p. 91-103. Disponible sur internet : http://www.recherches-en-education.net/IMG/pdf/REEno18.pdf (consulté le 30 janvier 2015).

BARRE-DE MINIAC C. (2000). Le rapport à l'écriture. Aspects théoriques et didactiques. Villeneuve d'Ascq. Université de Lille 3. Presses Universitaires du Septentrion.

BAYNE S. (2014). What's the matter with 'technology-enhanced learning'? Learning, Media and Technology. DOI:10.1080/17439884.2014.915851. Disponible sur internet : http://dx.doi.org/10.1080/17439884.2014.915851 (consulté le 29 juin 2014).

BERGE Z. L., MUILENBURG L. (2013). Handbook of mobile learning. Oxford, U.K. Routledge.

BOURQUE J., PRÉVOST N., LANG M. (2013). La Toile et la pensée critique : une conceptualisation deleuzienne. Revue STICEF, Vol. 20, p. 381-400. Disponible sur internet : $\quad$ http://sticef.univ-lemans.fr/num/vol2013/06-bourquereiah/sticef_2013_NS_bourque_06.htm (consulté le 30 janvier 2015).

BROUGERE G., BEZILLE H. (2007). De l'usage de la notion d'informel dans le champ de l'éducation. Revue française de pédagogie : recherches en éducation, Vol. 158, p. 117-160.

BROWN C., CZERNIEWICZ L. (2010). Debunking the digital native: beyond digital apartheid, towards digital democracy. Journal of Computer Assisted Learning, Vol. 26, p. 357-369.

BUCKINGHAM D. (2007). Beyond technology: Children's learning in the age of digital culture. Cambridge \& Malden MA. Polity Press.

CHARLIER B. (2014). Les Environnements Personnels d'Apprentissage: des instruments pour apprendre au-delà des frontières. Revue STICEF, Vol 21, $\mathrm{n}^{\circ}$ spécial «Les EPA: entre description et conceptualisation». Disponible sur internet: http://sticef.univ-lemans.fr/num/vol2014/10-charlierepa/sticef_2014_NS_charlier_10.htm (consulté le 30 janvier 2015).

CHEN W., SIU KAY CHOI A. (2011). Internet and social support among Chinese migrants in Singapore. New Media \& Society, Vol. 13, n 7, p. 1067-1084. 
CHRISTENSEN G., STEINMETZ B., ALCORN A., BENNET D., WOODS D., EMANUEL EJ. (2013). The MOOC Phenomenon: Who Takes Massive Open Online Courses and Why?. Social Science Research Network. Disponible sur internet : http://papers.ssrn.com/sol3/papers.cfm?abstract id=2350964 (consulté le 30 janvier 2015).

CLARK W., LOGAN K., LUCKIN R., MEE A., OLIVER M. (2009). Beyond Web 2.0: mapping the technology landscapes of young learners. Journal of Computer Assisted Learning, Vol. 25, p. 56-69.

COLLIN S. (2014). Saisir les usages numériques éducatifs des élèves dans leur globalité. Formation et profession, chronique Technologies en éducation, Vol. 21, n 2, p. 101-104.

COLLIN S., KARSENTI T. (2012a). Approches théoriques des usages des technologies en éducation : regard critique. Formation et profession, Vol. 20, n 3 , p. 60-72.

COLLIN S., KARSENTI T. (2012b). Using IT to Facilitate Linguistic Integration by Immigrants: An International Overview. Issues in Informing Science and Information Technology, Vol. 9, p. 243-251.

COLLIN S., KARSENTI T. (2013). Usages des technologies en éducation : analyse des enjeux socioculturels. Éducation et Francophonie, Vol. 16, nº 1, p. 192-210.

CONEIN B. (2004). Cognition distribuée, groupe social et technologie cognitive. Réseaux, Vol. 124, n 2, p. 53-79.

COOMBS P. H., AHMED M. (1974). Attacking Rural Poverty: How non-formal education can help. Baltimore. John Hopkins University Press.

COURSERA. (2014). Cours en ligne accessibles à tous et gratuits. https ://www.coursera.org/about/ (consulté le 24 juin 2014).

CRESWELL J. W. (2014). Research Design Qualitative, Quantitative, and Mixed Methods Approaches. Thousand Oaks, CA. SAGE Publications.

CUBAN L. (1986). Teachers and machines: The classroom use of technology since 1920. New York. Teachers College Press.

DAOUDI Y. (2011). Vers une intégration écologique des technologies de l'information et de la communication en éducation en Afrique du Centre et de l'Ouest: une approche culturelle. Thèse de doctorat inédite, Université de Montréal, Montréal.

DE HAAN J. (2004). A multifaceted dynamic model of the digital divide. IT \& Society, Vol. 1, n 7 , p. 66-88.

DIBAKANA J-A. (2010). NTIC et dynamiques sociales en Afrique : l'exemple du téléphone portable au Congo-Brazzaville. In A. Kiyindou (dir.), Cultures, technologies et mondialisation. Paris, France. Harmattan, pp. 25-36.

ENGESTRÖM Y. (2008). Weaving the texture of change. Journal of Educational Change Activity Theory and School Innovation, Vol. 9, $\mathrm{n}^{\circ}$ 4, p. 379-383.

ERSTAD O., ARNSETH H. C. (2013). Learning Lives Connected: Digital Youth across School and Community Spaces. Comunicar, Vol. 40, p. 89-98. Disponible sur internet : http://dx.doi.org10.3916/C40-2013-02-09 (consulté le 24 juin 2014).

ERUMBAN A., DE JONG S. (2006). Cross Country differences in ICT adoption: A consequence of culture? Journal of World Business, Vol. 41, $n^{\circ} 4, p$ 302314.

EYNON R. (2008). The learner and their context: review of the evidence. A report to BECTA. Oxford: Department of Education. University of Oxford.

EYNON R. (2012). The challenges and possibilities of the impact agenda. Learning, Media and Technology, Vol 37, n 1, p. 1-3. 
FAIRLIE R., ROBINSON J. (2013). Experimental Evidence on the Effects of Home Computers on Academic Achievement among Schoolchildren. American Economic Journal, vol. 5, $\mathrm{n}^{\circ}$ 3, p. 211-40. Press.

FEENBERG A. (1991). Critical theory of technology. New York. Oxford University

FEENBERG A. (2001). Whither Educational Technology? International Journal of Technology and Design Education, Vol. 11, n 1, p. 83-91.

FEENBERG, A. (2004). (Re)penser la technique. Vers une technologie démocratique. Trad. de l'anglais par A.-M. Dibon. Paris. La Découverte / M.A.U.S.S.

FEENBERG A. (2005). Critical theory of technology: An overview. Tailoring Biotechnologies, Vol. 1, n 1, p. 47-64.

FEENBerg A. (2014). Pour une théorie critique de la technique. Trad. de l'anglais par I . Arnaq et V. Dassas. Montréal. Lux/Humanités, 465p.

FLUCKIGER C. (2008). L'école à l'épreuve de la culture numérique des élèves. Revue Française de Pédagogie, Vol 163, n 1, p. 51-61.

FLUCKIGER C. (2011). La collaboration ordinaire des étudiants par les outils du web social. Colloque international EPAL, Grenoble, 23-25 juin 2011.

FLUCKIGER C. (2014). L'analyse des Environnements Personnels d'Apprentissage sous l'angle de la discontinuité instrumentale. Revue STICEF, Vol. 21. Disponible sur internet : http://sticef.univ-lemans.fr/num/vol2014/12-fluckigerepa/sticef 2014 NS fluckiger 12p.pdf (consulté le 29 janvier 2015).

FRIESEN N. (2008). Critical theory: Ideology critique and the myths of elearning. Ubiquity, Vol 2008 (June). Disponible sur internet : http://ubiquity.acm.org/article.cfm?id=1386860 (consulté le 29 janvier 2015).

FRIESEN N. (2013). Educational Technology and the New Language of Learning: Lineage and Limitations. In Selwyn N., Facer K. (dir.), The Politics of Education and Technology: Conflicts, Controversies, and Connections. New York; London. Palgrave, pp. 36-54.

FURLONG J., DAVIES C. (2012). Young people, new technologies and learning at home: Taking context seriously. Oxford Review of Education, Vol. 38, $\mathrm{n}^{\circ} 1$, p. 45 62.

GALLANT N., FRICHE C. (2012). Être ici et là-bas tout à la fois : réseaux sociaux en ligne et espaces d'appartenance chez les jeunes immigrants au Québec. Lien social et politiques, Vol. 64, p. 113-124.

GEORGE E. (2014). Quelles perspectives critiques pour aborder les TIC ?. Revue tic\&société, $\quad$ Vol. $\quad 8, \quad \mathrm{~N}^{\circ} \quad 1-2 . \quad$ Disponible sur internet : http://ticetsociete.revues.org/1365 (consulté le 29 janvier 2015)

GIRE F., GRANJON F. (2012). Les pratiques des écrans des jeunes Français. Déterminants sociaux et formes d'appropriation. Reset, vol. $1, \mathrm{n}^{\circ} 1$, Disponible sur internet : http://www.journal-reset.org/index.php/RESET/article/view/4/4 (consulté le 24 juin 2014).

GOUSETI A. (2010). Web 2.0 and education: Not just another case of hype, hope and disappointment? Learning, Media and Technology, Vol. 35, $\mathrm{n}^{\circ}$ 3, p. 351356.

GRANJON F. (2004). De quelques éléments programmatiques pour une sociologie critique des usages sociaux des TIC. In R. David (Dir.), actes du colloque Journée d'étude les rapports société-technique du point de vue des sciences de l'homme et de la société. Lares-Université de Rennes 2, pp. 1-6. Disponible sur internet: http://archivesic.ccsd.cnrs.fr/sic_00001136v2/ (consulté le 14 avril 2015). 
GRANJON F. (2009). Inégalités numériques et reconnaissance sociale. Les Cahiers du numérique, Vol.5, nº 1, p. 19-44.

GUICHON N. (2011). Apprentissage des langues médiatisé par les technologies: contribution à l'épistémologie de la didactique des langues. Note de synthèse en vue de l'obtention de l'Habilitation à Diriger des recherches. Université du Havre. Disponible sur internet : acedle.org/IMG/pdf/HDR_Nicolas_Guichon.pdf (consulté le 14 avril 2015).

GUICHON N. (2015). Quelle transition numérique pour les étudiants internationaux ? Alsic, Vol. 18. Disponible sur internet : http://alsic.revues.org/ (Consulté le 13 avril 2015).

HAMILTON E., FRIESEN N. (2013). Ethnology \& pedagogy: Autonomist \& instrumentalist configurations in scholarship. Canadian journal of learning and technology, Vol. 39, n² 2, p. 134-158.

HARGITTAI E. (2002). Second-level digital divide: Differences in people's online skills. First Monday, Vol. 7, $\mathrm{n}^{\circ} 4$. Disponible sur internet : http://firstmonday.org/issues/issue7 4/hargittai/index.htm (consulté le 24 juin 2014)

HARGITTAI E. (2010). Digital Na(t)ives? Variation in Internet Skills and Uses among Members of the «Net Generation ». Sociological Inquiry, Vol 80, $\mathrm{n}^{\circ} 1,92$ 113.

HELSPER E.J., EYNON R. (2010). Digital natives: where is the evidence? British Educational research Journal, Vol. 36, n 3, p. 503-520.

HENRI F., LUNDGREN-CAYROL K. (2001). Apprentissage collaboratif à distance: pour comprendre et concevoir les environnements d'apprentissage virtuels. Québec. Presse de l'Université du Québec.

HUTCHINS E. (1995). Cognition in the Wild. Cambridge, MA. MIT Press.

HUTCHINS E. (2000). Distributed cognition. International Encyclopedia of the Social and Behavioral Sciences. Oxford, U.K. Elsevier Science. Disponible sur internet: http://files.meetup.com/410989/DistributedCognition.pdf (consulté en 29 janvier 2015).

ITO M., BAUMER S., BITTANTI M., BOYD D., CODY R., HERR-STEPHENSON B., HORST H.A., LANGE P.G., MAHENDRAN D., MARTINEZ K.Z., PASCOE C.J., PERKEL D., ROBINSON L., SIMS C., TRIPP L. (2010). Hanging out, messing around and geeking out. Kids living and learning with new media. Cambrige, MA. MIT Press. Disponible sur internet: $\mathrm{http}: / /$ mitpress.mit.edu/books/hanging-out-messingaround-and-geeking-out (consulté le 24 juin 2014).

ITO M., GUTIERREZ K., LIVINGSTONE S., PENUEL B., RHODES J., SALEN K., SCHOR J., SEFTON-GREEN J, WATKINS S.C. (2013). Connected Learning: An Agenda for Research and Design. Technical Report, 99p. Irvine, CA. Digital Media and Learning Research Hub.

KHARBECHE T. (2006). L'impact des facteurs socioculturels dans l'adoption des TIC en Afrique du Nord : état de l'art et enjeux. In C. Ammi (dir.), Innovations technologiques : aspects culturels et mondialisation. Paris, France. Lavoisier, pp. 75-90.

KOLOWICH, S. (2013). Coursera Snags \$43-Million in Venture Capital. The Chronicle of Higher Education. Disponible sur internet : http://chronicle.com/blogs/wiredcampus/mooc-company-snags-43-million-inventure-capital/44667 (consulté le 25 juin 2014).

KLUZER S., FERRARI A., CENTENO C. (2009). ICT for Learning the Host Country's Language by Adult Immigrants in the EU. Sevilla, Spain. JRC Institute for Prospective Technological Studies, European Commission. 
KUMPULAINEN K., SEFTON-GREEN J. (2014). What is connected learning and how to research it? International Journal of Learning and Media, Vol. 4, $\mathrm{n}^{\circ} 2$, p. 7-18.

LAI K.-W., KHADDAGE F., KNEZEK G. (2013). Blending student technology experiences in formal and informal learning. Journal of Computer Assisted Learning, Vol. 29, n 2, p. 414-425.

LANGOUËT G. (2000). Les inégalités entre États et populations de la planète. Paris. L'Harmattan.

LAURILLARD D. (2014). What is the problem for which MOOCs are the solution? IOE London blog. Disponible sur internet : http://ioelondonblog.wordpress.com/2014/05/14/what-is-the-problem-for-whichmoocs-are-the-solution/ (consulté le 25 juin 2014).

LEANDER K., PHILLIPS N. C., TAYLOR K. H. (2010). The changing social spaces of learning: Mapping new mobilities. Review of Research in Education, Vol. 34, $\mathrm{n}^{\circ} 1$, p. 329-394.

LEASK M. (2011). Improving the professional knowledge base for education: Using knowledge management and Web 2.0 tools. Policy Futures in Education, Vol. 9, n 5, p. 644-660.

LIVINGSTONE D. W. (2001). Adults informal learning: Definitions, finds, gaps, and future research: New approaches for lifelong learning. Toronto. Ontario Institute for Studies in Education.

LIVINGSTONE S., HELSPER E. (2007). Gradations in digital inclusion: Children, young people and the digital divide. New Media \& Society, Vol. 9, n 4, p. 671-696.

MALE T., BURDEN K. (2013). Access denied? Twenty-first-century technology in schools. Technology, Pedagogy and Education. DOI:10.1080/1475939X.2013.864697

MATTELART T. (2009). Les diasporas à l'heure des technologies de l'information et de la communication : petit état des savoirs. Revue tic\&société, Vol. $3, n^{\circ} 1-2$, p.35-48.

MADDUX C. D., JOHNSON D. L. (2012). External validity and research in information technology in education. Computers in the Schools, Vol. 29, $\mathrm{n}^{\circ} 3, \mathrm{p}$. 249-252.

MESCH G., TALMUD I. (2011). Wired youth: The social world of adolescence in the information age. Oxford. Routledge.

MILLERAND F. (2002). La dimension cognitive de l'appropriation des artefacts communicationnels. In F. Jauréguiberry et S. Proulx (dir.), Internet : nouvel espace citoyen. Paris. L'Harmattan, pp. 181-203.

MILLERAND F. (2003). L'appropriation du courrier électronique en tant que technologie cognitive chez les enseignants chercheurs universitaires : Vers l'émergence d'une culture numérique. Thèse en communication, Faculté des Arts et des Sciences, Université de Montréal.

MOCKER D. W., SPEAR G. E. (1982). Lifelong Learning: Formal, Nonformal, Informal, and Self-Directed. Information Series, Vol 241, $\mathrm{N}^{\circ}$ 134-159.

NISTOR N., LERCHE T., WEINBERGER A., CEOBANU C. HEYMANN J. O. (2014). Towards the integration of culture in the Unified Theory of Acceptance and Use of Technology. British Journal of Educational Technology, Vol. 45, $\mathrm{n}^{\circ} 1$, p. 36-55.

NORMAN D. (1998). The Invisible Computer. Cambridge. MIT Press.

NUNN J., KADEL R., EATON-KAWECKI KARPYN A. (2002). A digital divide in Maryland public schools. The Electronic Journal of Communication/La revue Electronic 
de communication, Vol. 12. Disponible sur internet : http://www.cios.org/EJCPUBLIC/012/1/01214.html (consulté le 24 juin 2014).

PENLOUP M.-C. (1999). L'écriture extrascolaire des collégiens, Des constats aux perspectives didactiques. Clio. Histoire, femmes et sociétés, Vol 11. Disponible sur

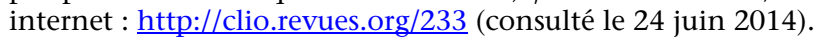

OCDE. (2010). Are the New Millenium Learners Making the Grade? Technology Use and Educational Performance in PISA. Paris, OCDE.

OCDE. (2011). L'enseignement supérieur à l'horizon 2030. Paris. OCDE.

RAFALOW M. H. (2014). The Digital Divide in Classroom Technology Use: A Comparison of Three Schools. International Journal of Sociology of Education, Vol. 3, $\mathrm{n}^{\circ} 1, \mathrm{p} .67-100$.

SCHRADIE J. (2012). The Trend of Class, Race, and Ethnicity in Social Media Inequality. Information, Communication \& Society, Vol. 15, $n^{\circ} 4$, p. 555-571.

SCHNEIDER É. (2013). Économie scripturale des adolescents : enquête sur les usages de l'écrit de lycéens. Thèse de doctorat. Caen. Université de Caen, disponible sur internet :http://tel.archives-ouvertes.fr/tel-00911228 (consulté le 30 janvier 2015).

SELWYN N. (2010). Looking beyond learning: Notes towards the critical study of educational technology. Journal of Computer Assisted Learning, Vol. 26, n 1, p. 65-73.

SPOTTI M., KLUZER S., FERRARI A. (2010). ICT for L2 Acquisition by Adult Migrants: Results from a Comparative Study in the Netherlands and Sweden. Paper presented at the ICT for Language Learning - 3rd Edition. Disponible sur internet : http://pixelonline.org/ICT4LL2010/common/download/Proceedings pdf/ ILL01-Spotti,Kluzer,Ferrari.pdf (consulté le 24 juin 2014).

TEDDLIE C., TASHAKKORI A. (2009). Foundations of Mixed Methods Research. Integrating Quantitative and Qualitative Approaches in the Social and Behavioral Sciences. Sage. Thousand Oaks, CA.

THORNE S., FISCHER I., LU X. (2012). The semiotic ecology and linguistic complexity of an online game world. ReCALL, Vol. 24, n' 3 , p. 279-301.

UNGERLEIDER C. (2002). Information and communication technologies in elementary and secondary education: A state of the art review. Actes du Colloque 2002 du Programme pancanadien de recherche en éducation (PPRE) : La technologie de l'information et l'apprentissage. Disponible sur internet: http://www.cemec.ca (consulté le 29 janvier 2015).

UNDERWOOD J., DILLON G. (2011). Chasing dreams and recognising realities: Teachers' responses to ICT. Technology, Pedagogy and Education, Vol. 20, $\mathrm{n}^{\circ} 3$, p. 317-330.

UNESCO (2011). TIC UNESCO : Un référentiel de compétences pour les enseignants. Paris: UNESCO. Disponible sur internet : http://unesdoc.unesco.org/images/0021/002169/216910f.pdf

WARSCHAUER M. (1998). Online learning in sociocultural context. Anthropology and Education Quarterly, Vol. 29, $\mathrm{n}^{\circ} 3$, p. 68-88.

WEI L. (2012). Number Matters: The Multimodality of Internet Use as an Indicator of the Digital Inequalities. Journal of Computer-Mediated Communication, Vol. 17, n 2, p. 303-318. 